\title{
Laparoscopic Trainer with Pneumoperitoneum
}

\author{
José L. Ortiz Simón ${ }^{1}$, Arturo Minor Martínez ${ }^{2}$, Neftalí Prado Coronado ${ }^{2}$, Ricardo Ordorica Flores ${ }^{3}$ \\ ${ }^{1}$ Department of Electrical and Electronics Engineering, Technological Institute of Nuevo Laredo, Nuevo Laredo, México \\ ${ }^{2}$ Bioelectronics Section, Center for Research and Advanced Studies of the IPN, México DF, México \\ ${ }^{3}$ Pediatrics Section, Federico Gómez Children's Hospital, México DF, México \\ Email: jlortiz@cinvestav.mx, aminor@cinvestav.mx, karmariv@hotmail.com,ricaordor@hotmail.com
}

Received May 12, 2012; revised June 15, 2012; accepted July 3, 2012

\begin{abstract}
The development of skills and abilities in laparoscopy is directly related to the use of trainers. The trainer should model the patient with all its natural complexities as closely as possible. In this article we propose a system for training the establishment of pneumoperitoneum executing the basic tasks to create the workspace in the trainer, the insertion of primary umbilical trocar and simulate some of the everyday problems in surgery. Materials and Methods: A group of electrical with biomedical engineers and laparoscopic surgeons developed a physical trainer that allows implementing the pneumoperitoneum. The system uses pneumatic electro valves that are controlled with a dedicated microprocessor. The user can program the system to set the parameters of the pneumoperitoneum. Results: This new trainer facilitated the programming of right values of parameters to distend the abdominal cavity according a specific clinical case. The model developed enables the trainee to consolidate his knowledge on establishing the parameters required within clinical practice, as well as the entry techniques. Conclusions: A new physical model for laparoscopic training was designed. The system enables the laparoscopic surgeon to set the parameters for establishing the workspace according to the clinical case. The trainer allows the surgeon to train in the Hasson technique for the introduction of the first trocar, as well as the placement of the rest of surgical instruments with video assistance. We think this new trainer system will help minimize entry-related injuries.
\end{abstract}

Keywords: Laparoscopy; Trainer; Pneumoperitoneum

\section{Introduction}

Laparoscopic trainers enable surgeons to develop their skills and consolidate new methodologies in solving surgical problems [1]. From the appearance of the first physical trainers, until the latest generation of trainers both hybrid and virtual [2], the surgeon has been reinforcing his entire knowledge and developing the abilities necessary for carrying out routine procedures with tools which are explicitly developed to simulate problems that occur in the operating room [3]. Additionally, throughout the whole process of finding the best training methodology, it has been demonstrated that integrating virtual and real training is the best method for achieving training objectives [4]. The establishment of the pneumoperitoneum has been neglected in the majority of trainers conceived solely as a workspace. The reality is that this requires a variety of important criteria, in accordance with the clinical symptoms of the patient [5]. We consider the surgeon should consolidate his technical knowledge as part of his surgical practice, since more than 50\% of greater complications in laparoscopy take place in this stage of the procedure [6]. It is common to find errors resulting from lack of skills in surgical practice during initial entry to establish the pneumoperitoneum [7], such as the exaggerated perforation of the entrances with the oft-repeated correction on carrying out knots around the trocar. It is also common to find a lack of adequate knowledge for the establishing of pneumoperitoneum in a newborn infant. Surgeons may confuse the exact entry point of the trocars between a baby patient and an adult, besides the risks of abdominal pressurization [8]. For this reason, we propose a new trainer for laparoscopy with the capacity to generate the pneumoperitoneum with the different clinical variables that may arise in the operating room.

\section{Materials and Methods}

The pneumoperitoneum is the workspace of a laparoscopic surgeon. It is based on the introduction of $\mathrm{CO}_{2}$ gas at a certain pressure and flow, through the main entry port through which enters the laparoscope [9].

The pressure and flow required to generate the pneumoperitoneum depend on variables such as the type of operation, the position, the age and weight of the patient $[10,11]$. Due to this, it is important that the laparoscopic surgeon knows the clinical case perfectly. This new trainer 
uses pressured air to eliminate the unnecessary consumption of $\mathrm{CO}_{2}$. The basic parameters used to establish the pneumoperitoneum are listed on Table 1, according to the clinic case of the patient [12].

The system is build with a microcontroller that uses the data of a pressure sensor to control the inlet valve of air pumped into the cavity. Figure 1 shows the block diagram of the electronic system implemented. The correct pressure and flow parameters for the pneumoperitoneum the required values are programmed through a control panel. The outlet valve allows fine control of air. It allows complete venting of gases produced by electrocauterization minimizing the lack of visibility. The values programmed into the system are calibrated in millimeters of mercury.

\section{Tests}

A soccer ball inner bladder, made of low density neoprene and $3 \mathrm{~mm}$ thickness, was placed in a child mannequin. It allows the skin of the trainer to be flexible and can be lifted up as with human skin. It adjusts to the trocar size and can be sutured. An air compressor was used as a source of gas. A zero degree optic laparoscope was used as a source of video and a commercial television set as a monitor. The new trainer was tested with a specialized

Table 1. Parameters for establishment of the pneumoperitoneum.

\begin{tabular}{ccc}
\hline Age & Pressure & Flow \\
\hline New born & $5 \mathrm{mmHg}$ & $0.5 \mathrm{lt}$ \\
Infant $<$ & $5-7 \mathrm{mmHg}$ & $1.0 \mathrm{lt}$ \\
$\begin{array}{c}\text { Infant }> \\
\text { Pre-school aged } \\
\text { children }\end{array}$ & $7-10 \mathrm{mmHg}$ & $2.0 \mathrm{lt}$ \\
$\begin{array}{c}\text { School aged } \\
\text { children } \\
\text { Adolescents }\end{array}$ & $10-12 \mathrm{mmHg}$ & $3.0 \mathrm{lt}$ \\
Adults & $10-13 \mathrm{mmHg}$ & $4.0 \mathrm{lt}$ \\
\hline
\end{tabular}

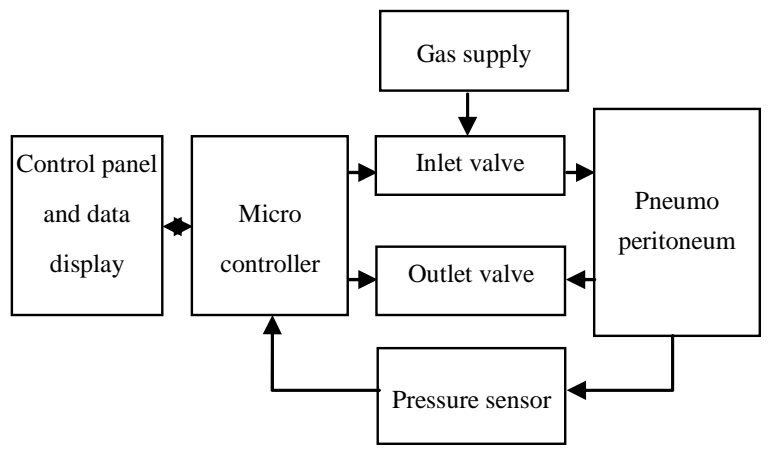

Figure 1. Block diagram of the electronic pressure control system. surgeon, carrying out puncture with the Hasson technique. The pressure control system was programmed considering a normal clinical case of a 3-year-old child for laparoscopic cholecystectomy procedure. The suggested values were $6 \mathrm{mmHg}$ and $1.0 \mathrm{~L}$ [12]. The first trocar was introduced under direct vision. The trainer was insufflated and a surrounding exploration was carried out. After that, two trocars were inserted with the assistance of the endocamera. The system was tested simulating a different clinical case: a 15 years old adolescent with diagnosed Appendicitis. The suggested parameters to establish the pneumoperitoneum were set at $11 \mathrm{mmHg}$ gas pressure and $4 \mathrm{~L}$ as a maximum flow. A Veress needle insertion following the "safe insertion technique" allowed access to the peritoneal cavity (Figure 2(a)) [13].

After inserted the Veress needle, air was pumped up to $7 \mathrm{mmHg}$ to create the workspace and verify there were no air leaks. After that, the pressure was increased to $11 \mathrm{mmHg}$ and a brief surrounding exploration was made. Two more $5 \mathrm{~mm}$ trocars were placed in the usual position to get the configuration of three entrances for appendicectomy [14] with the assistance of the laparoscope (Figure 2(b)). Finally, a cutting task was performed inside the pressurized chamber. The task consisted of cutting a circle of the chamber wall without perforating the exterior layer simulating a dissection (Figure 3(a)). The trainer allows adding material or tasks to work with. The intraperitoneal air pressure was maintained to $11 \mathrm{mmHg}$ throughout the task execution. An expert surgeon carried out the task with no time restriction. Securing of the trocar and knot techniques were also practiced in order to keep the trocar fixed during the introduction and extraction of the instrument (Figure 3(b)).

Different training exercises were carried out such transfer, cutting and suturing tasks inside the pressurized work space. An animal model dissection was performed as well (Figure 4).

\section{Discussion}

Trainers for laparoscopic surgery do not facilitate training of all of the necessary maneuvers of a real surgery [15]. The abilities of the laparoscopic surgeon should be complemented by small tricks, which enable them to resolve real problems in the operating room. The creation of the pneumoperitoneum is a critical stage; it requires a priori knowledge of the technology and methods implied to achieve it. The pneumoperitoneum is one of the first obstacles the new surgeon is going to be confronted with. Current trainers do not contemplate the establishment of the work space parameters with the functional conditions of the patient. The introduction of the first trocar and maintaining the variables of the pneumoperitoneum within 


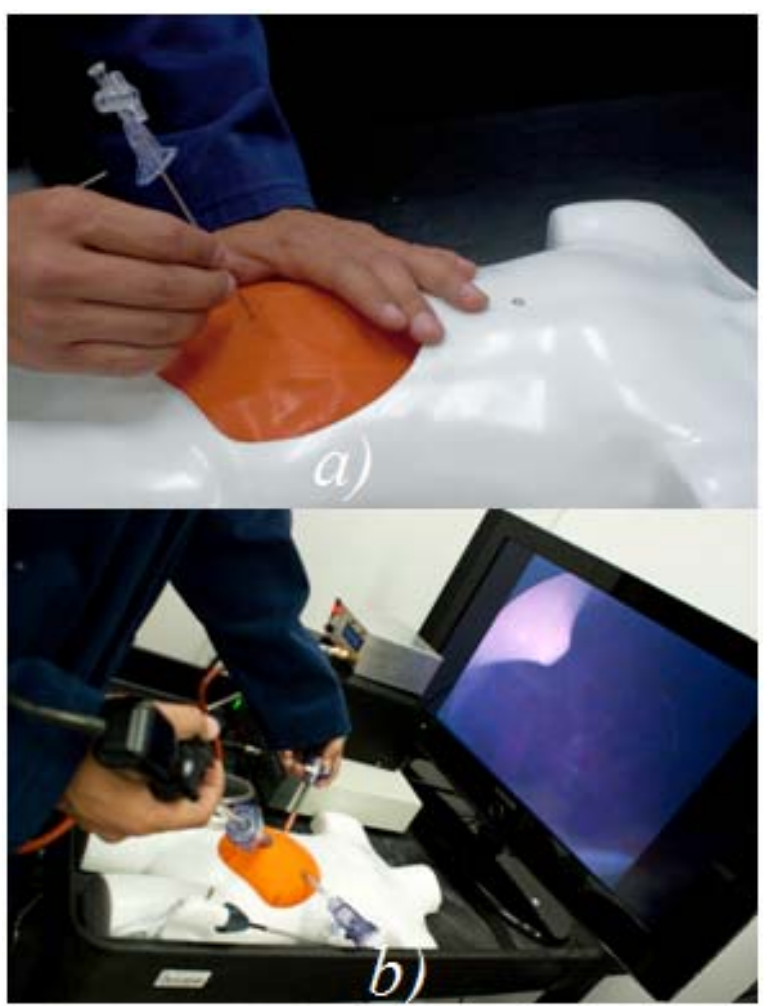

Figure 2. (a) Puncture with a Veress needle; (b) Insertion of trocars with video assistance.

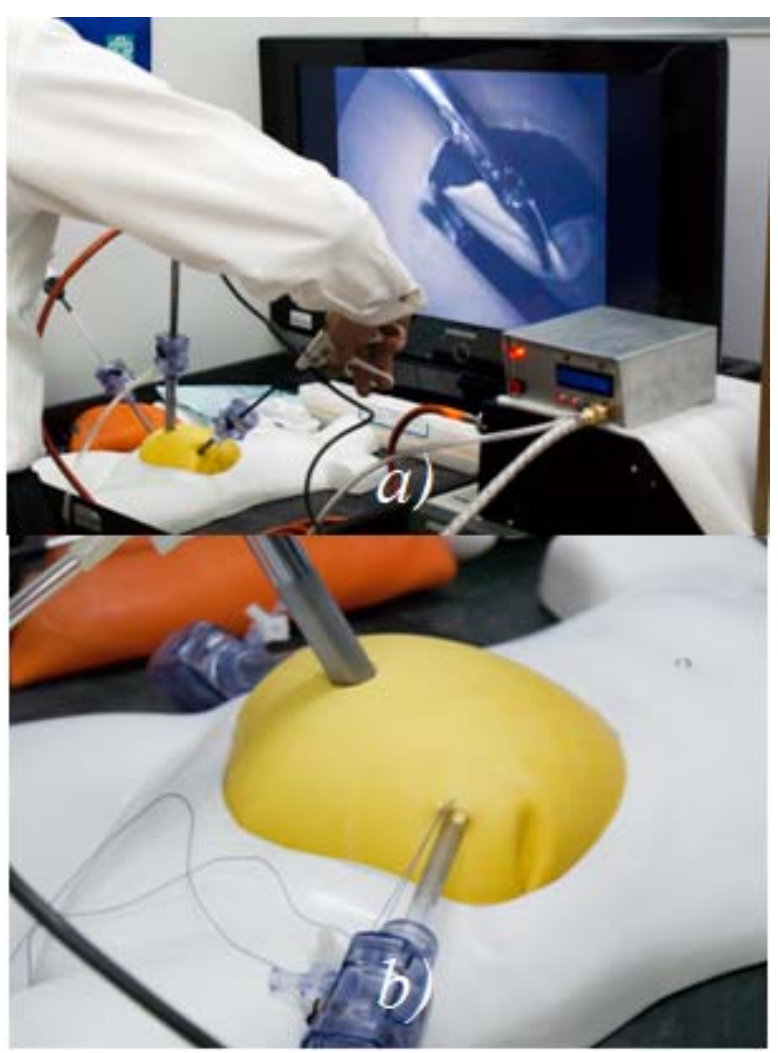

Figure 3. (a) Cutting task inside the pressurized chamber; (b) Suture to fix the trocar to the proper height.

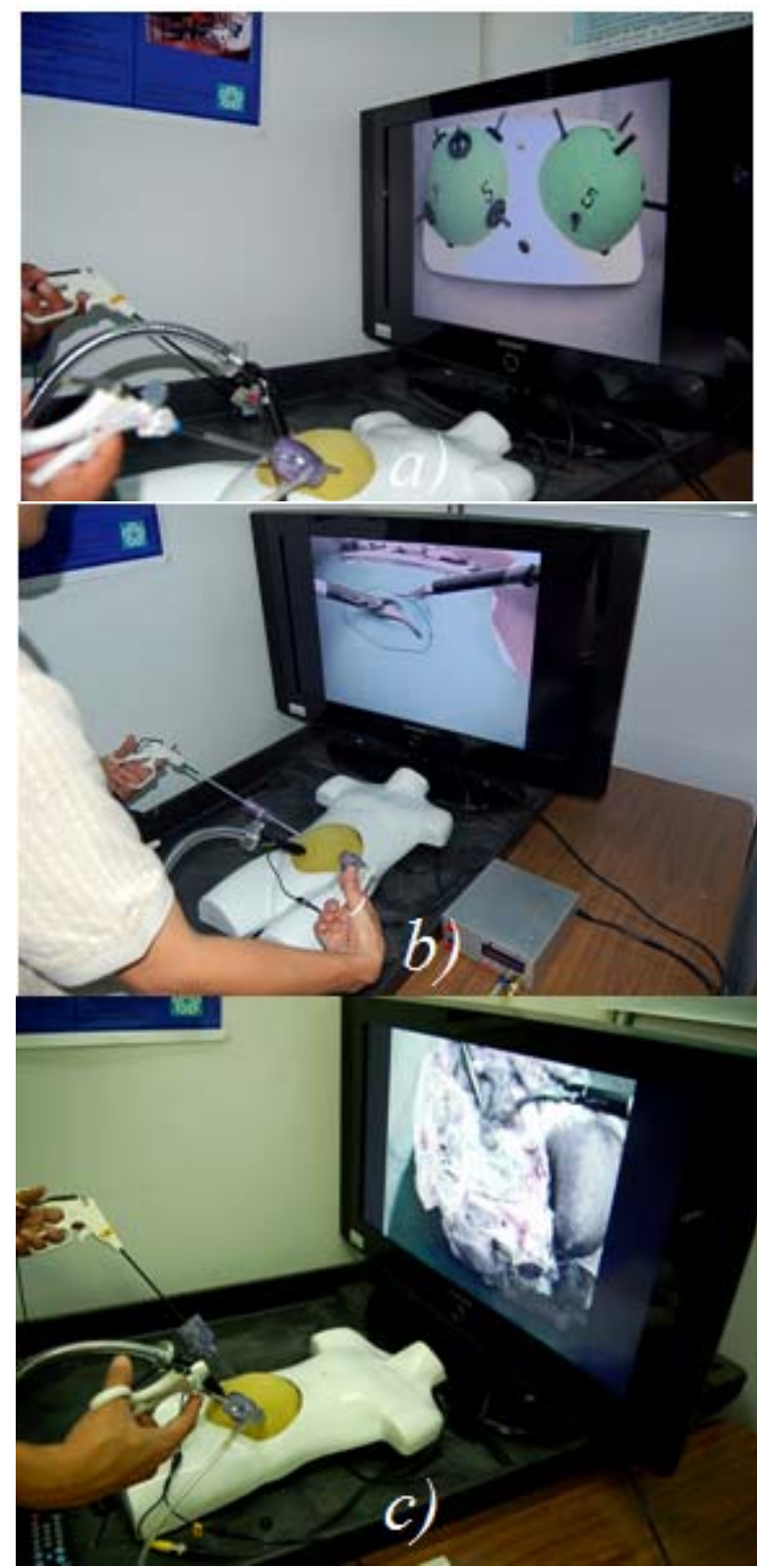

Figure 4. Tasks inside the pressurized chamber: a) Transferring rubber rings; b) MISTELS cutting task modality; c) Animal model dissecting.

safe ranges are not considered because this possibility does not exist in current trainers.

The present design of trainer teaches to program the necessary parameters for establishing the pneumoperitoneum according to the particular clinical case. It allows the novice surgeon to perform the maneuver of inserting the first trocar under direct vision with the Hasson technique. This trainer system accustoms the surgeon carry out the initial surrounding exploration. Besides, the trainer allows him to introduce the rest of the trocars with video-assistance and with the implicit knowledge of the surgical technique to apply. Additionally, this trainer allows surgeons put tricks 
into practice for avoiding gas leaks caused by the widening out of entry ports. We have used the same bladder up to 10 times successfully. This novel trainer will allow including critical variables of intra-abdominal pressure during practice sessions in a dynamic and realistic way. This trainer will certainly allow establish a one more step to the training tasks collection that contributes to enhance the quality of newly graduated laparoscopic surgeons.

\section{Conclusion}

We developed the first physical trainer with pneumoperitoneum. This novel trainer facilitates the integration of the knowledge of the surgeon, based on the clinical conditions of the patient. It allows the surgeon to practice the Hasson technique, the safe insertion of the Veress needle through an incision beneath the umbilical scar while the abdominal wall is lifted with Alice forceps. We tried basic laparoscopy MISTEL's tasks that we do not see any problem to simulate perivisceral laparoscopy tasks. Entry related injuries such perforation of subsurface blood vessels will be reduced in the operating room with the use of this system to train new laparoscopic surgeons.

\section{REFERENCES}

[1] R. Katz, "Methods of Training Using Pelvic Trainers," Current Urology Reports, Vol. 7, No. 2, 2006, pp. 100106. doi:10.1007/s11934-006-0067-0

[2] M. P. Laguna, H. Wijkstra, "Volume 11: Training in Laparoscopy,” In: J. J. M. C. H. de la Rosette and I. S. Gill, Eds., Laparoscopic Urologic Surgery in Malignancies, Springer, New York, 2005, pp. 260-261.

[3] M. P. Laguna, T. M. de Reijke and J. J. de la Rosette, "How Far Will Simulators be Involved into Training?" Current Urology Reports, Vol. 10, No. 2, 2009, pp. 97105. doi:10.1007/s11934-009-0019-6

[4] K. Madan and C. T. Frantzides. "Prospective Randomized Controlled Trial of Laparoscopic Trainers for Basic Laparoscopic Skills Acquisition,” Surgical Endoscopy, Vol. 21, No. 2, 2007, pp. 209-213. doi:10.1007/s00464-006-0149-6

[5] J. Schleef, "Chapter 8: Complications of Endoscopic Sur- gery in Infants and Children,” In: Endoscopic Surgery in Infants and Children, Springer, New York, 2008, pp. 6667.

[6] G. A. Vilos, “The ABCs of a Safer Laparoscopic Entry,” Journal of Minimally Invasive Gynecology, Vol. 13, No. 3, pp. 249-251. doi:10.1016/j.jmig.2005.12.005

[7] G. A. Vilos, A. G. Vilos, B. Abu-Rafea, J. Hollett-Caines, Z. Nikkhah-Abyaneh and F. Edris, "Three Simple Steps during Closed Laparoscopic Entry May Minimize Major Injuries,” Surgical Endoscopy, Vol. 23, No. 4, 2008, 758764 .

[8] T. Fujimoto, O. Segawa, G. J. Lane, S. Esaki and T. Miyano, "Laparoscopic Surgery in Newborn Infants," Surgical Endoscopy, Vol. 13, No. 8, 1999, 773-777. doi:10.1007/s004649901096

[9] T. N. Pappas and A. M. Fecher. "Principles of Minimally Invasive Surgery,” Surgery, Sec. 4, 2008, pp. 771-790.

[10] D. S. Beebe, S. Zhu, M. V. S. Kumar, V. Komanduri, J. A. Reichert and K. G. Belani, "The Effect of Insufflation Pressure on $\mathrm{CO}_{2}$ Pneumoperitoneum and Embolism in Piglets,” Anesthesia \& Analgesia, Vol. 94, No. 5, 2002, pp. 1182-1187. doi:10.1097/00000539-200205000-00024

[11] C.-G. Schmedt, O. Heupel, V. Riemer and C. N. Gutt, "Insufflation Profile and Body Position Influence Portal Venous Blood Flow during Pneumoperitoneum,” Surgical Endoscopy, Vol. 17, No. 12, 2003, pp. 1951-1957. doi:10.1007/s00464-002-9244-5

[12] A. K. Saxena, “Chapter 6: Effects of insufflation,” In: A. K. Saxena and M. E. Höllwarth, Eds., Essentials of Pediatric Endoscopic Surgery, Springer, New York, 2008, pp. 59-62.

[13] T. Cakir, D. Tuney, S. Esmaeilzadem and A. O. Aktan, "Safe Veress Needle Insertion," Journal of Hepato-Biliary-Pancreatic Surgery, Vol. 13, No. 3, 2006, pp. 225227. doi:10.1007/s00534-005-1024-X

[14] H. Steyaert and J. S. Valla. "Chapter 47: Laparoscopic Appendectomy in Children.” In: A. K. Saxena and M. E. Höllwarth, Eds., Essentials of Pediatric Endoscopic Surgery, Springer, New York, 2008, pp. 342-343.

[15] A. K. Bell, M. Zhou, S. D. Schwaitzberg and C. G. L. Cao, "Using a Dynamic Training Environment to Acquire Laparoscopic Surgery Skill,” Surgical Endoscopy, Vol. 23, No. 10, 2009, pp. 2356-2363. doi:10.1007/s00464-009-0346-1 